\title{
Properties and application of ultrasonic Lamb waves in $\mathrm{Cd}_{\mathrm{x}} \mathrm{Hg}_{1-\mathrm{x}} \mathrm{Te}$ plates
}

\author{
I.O. Lysiuk, V.F. Machulin, Ja.M. Olikh \\ Institute of Semiconductor Physics, NAS of Ukraine, 45 prospect Nauky, 03028 Kyiv, Ukraine \\ Phone: 380 (044) 265 6256; fax: 380 (044) 265 8342; e-mail: olikh@class.semicond.kiev.ua
}

\begin{abstract}
Group and phase velocities of the lowest orders of Lamb waves in $<100>,<110>$ directions for $(100) \mathrm{Cd}_{0.2} \mathrm{Hg}_{0.8} \mathrm{Te}$ plates are calculated. Frequency dispersion of $a_{0}$ and $s_{0}$ Lamb modes velocities were measured on (111)-plates in the range of frequencies from 0.2 to 10 $\mathrm{MHz}$. The frequency dependencies of relative efficiency of the Lamb modes excitation using two geometrical versions: symmetrical - with two piezoelectric transducers, and antisymmetrical with one piezoelectric transducer, have been studied. Possible applications of studied waves including ultrasonic treatment of semiconductors have been discussed.
\end{abstract}

Keywords: $\mathrm{Cd}_{\mathrm{x}} \mathrm{Hg}_{1-\mathrm{x}} \mathrm{Te}$ solid solutions, ultrasonic treatment, Lamb waves, efficiency of ultrasonic modegeneration.

Paper received 15.01.02; revised manuscript received 25.02.02; accepted for publication 05.03.02.

\section{Introduction}

Taking into account low structural perfection of $\mathrm{Cd}_{\mathrm{X}} \mathrm{Hg}_{1-\mathrm{X}} \mathrm{Te}$ solid solutions including blocks, high dislocation density, impurity and clusters, it is useful to apply intense ultrasonic waves for improving their structure $[1,2]$. Usage of ultrasonic waves during manufacturing semiconductor devices allows to create new devices with controlled characteristics [3]. Improvement of parameters of $\mathrm{Cd}_{\mathrm{X}} \mathrm{Hg}_{1-\mathrm{X}} \mathrm{Te}$ chips was reached with treatment by bulk waves [1-3]. But from the other point of view, allowance for plate geometry of bulk crystals using plate waves seems more suitable for treatment of such crystals. The properties of plate waves called normal waves in plate (NWP) is much more various than bulk waves, so they can find more wide applications including design of acousto-electronic sensors [4]. NWP are characterized by a great dispersion of phase and group velocity of propagation, complicated distribution of elastic deformation along thickness, frequency intervals where certain mode exists. Because of simple, method for generation of such waves in piezoelectric $\mathrm{A}_{2} \mathrm{~B}_{6}$ compounds was carefully studied [5]. A number of physical results on influencing ultrasonic waves on properties of semiconductors are obtained on non-piezoelectrics $[5,6]$.
Let's pay attention to the fact that the applied dynamical ultrasonic treatment of semiconductor devices in technological processes has some difficulties because of limited access space, high requirements to uniformity of deformation fields in the bulk or surface.

In order to show an advantage of used Lamb waves for ultrasonic treatments of $\mathrm{Cd}_{\mathrm{X}} \mathrm{Hg}_{1-\mathrm{X}} \mathrm{Te}$ crystals, let's have a brief review of mechanism of ultrasonic interaction with defects. The crystals have a high density of dislocations $\left(10^{4}-10^{6} \mathrm{~cm}^{-2}\right)$ and point defects (more than $10^{15} \mathrm{~cm}^{-3}$ ). When a dislocation is in ultrasonic field stress with sufficient intensity, it can oscillate with enormous (macroscopic) ampitude [7]. There are possible processes of redistribution of nonequilibrium points defects in the crystal bulk and their recombination during oscillations of a dislocation, which cause an improvement of electrophysical parameters of the crystal [1,2]. Maximum of a dislocation oscillation amplitude is effected in geometry, when vector of deformation is perpendicular to dislocation line. Therefore, ultrasonic treatment of semiconductor specimen with bulk or SH normal waves, which have only one direction of deformation, involves only part of dislocation into oscillatons and take part in redistribution, recombination process of defects. In the case of ultrasonic treatment with Lamb waves that have an elliptic 


\section{I.O.Lysiuk et al.: Properties and application of ultrasonic Lamb waves...}

polarisation, an arbitrary oriented dislocation can oscillate. Thus, the type of treatment enables to increase the number of dislocations involved into the process of redistribution and recombination of defects.

The purpose of the work is to analyze elastic properties for Lamb waves as particular case of NWP in $\mathrm{Cd}_{\mathrm{X}} \mathrm{Hg}_{1-\mathrm{X}} \mathrm{Te}$ plates in general and to study possible applications of certain modes.

\section{Normal waves in $\mathrm{Cd}_{0.2} \mathrm{Hg}_{0.8}$ Te plates}

The ultrasonic waves in a plate crystal with free boundaries are determined by the following equation and edge conditions:

$$
\begin{gathered}
\rho \ddot{U}_{i}=C_{i j n l} \frac{\partial^{2} U l}{\partial x_{i} \partial x_{n}} \\
T_{i j} n_{j}=0
\end{gathered}
$$

where $U_{i}$ - deformation along direction $x_{i}, C_{i j n l}$ elastic modulus, $T_{i j}$-stresses, $n_{i}$ - unit vector along $x_{i}$ direction, $\rho$ - density. Dispersion curves, which define the velocity of NWP various modes as a function of frequency, can be determined by solving the elastic equations of motion (1) and edge condition (2), as discussed by Victorov [8] and Tursunov [9].

In case of peculiar planes and directions of propagation, the system of equations (1), (2) can be converted into two independent systems. The first of these systems describes transversal normal waves (TNWP); the second does Lamb waves (LW). Each of these two types of waves consists of two groups: symmetrical and antisymmetrical. The division connected with symmetry of deformation with respect to median plate. Equations obtained from the system of equations (1) and (2) for the Lamb wave in (100) cube crystal plate along $<100>$ and $<110>$ direction of propagation are as follows [9]:

for symmetric waves:

$$
\frac{\operatorname{tg} \beta_{2} q h}{\operatorname{tg} \beta_{1} q h}=\frac{\left(\gamma_{1}+\beta_{1}\right)\left(c_{12}+c_{11} \beta_{2} \gamma_{2}\right)}{\left(\gamma_{2}+\beta_{2}\right)\left(c_{12}+c_{11} \beta_{1} \gamma_{1}\right)}
$$

for antisymmetric waves:

$$
\frac{\operatorname{tg} \beta_{2} q h}{\operatorname{tg} \beta_{1} q h}=\frac{\left(\gamma_{2}+\beta_{2}\right)\left(c_{12}+c_{11} \beta_{1} \gamma_{1}\right)}{\left(\gamma_{1}+\beta_{1}\right)\left(c_{12}+c_{11} \beta_{2} \gamma_{2}\right)}
$$

where, $q v=2 \pi f=w, q$-wave vector, $V$-phase velocity of propagation.

$$
\gamma_{1,2}=\frac{\beta_{1,2}\left(c_{12}+c_{44}\right)}{\rho V^{2}-c_{11} \beta_{1,2}^{2}-c_{44}}
$$

For $<100>$ direction: $\beta_{1,2}^{2}=A_{1} \pm \sqrt{A_{1}^{2}-B_{1}}$, where

$$
A_{1}=\frac{\left(c_{11}+c_{44}\right) \rho v^{2}+c_{12}\left(c_{12}+2 c_{44}\right)-c_{11}^{2}}{2 c_{11} c_{44}}
$$

$B_{1}=\frac{\rho^{2} v^{4}-\left(c_{11}+c_{44}\right) \rho v^{2}}{c_{11} c_{44}}$

for $<110>$ direction: $\beta_{1,2}^{2}=A_{2} \pm \sqrt{A_{2}^{2}-B_{2}}$,

where

$$
\begin{aligned}
& A_{2}=\frac{1}{2 c_{11} c_{44}}\left[\left(c_{11}+c_{44}\right) \rho v^{2}+c_{12}\left(c_{12}+2 c_{44}\right)-\frac{c_{11}}{2}\left(c_{11}+c_{12}+2 c_{44}\right)\right] \\
& B_{2}=\frac{1}{c_{11} c_{44}}\left[\rho^{2} v^{4}-\left(\frac{c_{11}+4 c_{44}+c_{12}}{2}\right) \rho v^{2}+\frac{c_{44}\left(c_{11}+c_{12}+2 c_{44}\right)}{2}\right]
\end{aligned}
$$

Group velocity is calculated by the formula

$$
V_{\mathrm{gr}}=\frac{\partial \omega}{\partial v}=\frac{V_{\mathrm{ph}}}{1-\frac{\omega}{V_{\mathrm{ph}}} \frac{\partial V_{\mathrm{ph}}}{\partial \omega}}
$$

Equations (3), (4) are transcendental, and their solution cannot be found as an analytical function $v$ from $\omega$. The problem has additional difficulties for solving in some phase velocity intervals when the equations transform into the complex ones. In the work, roots of equations (3), (4) were found by means of finding minima of functions

$$
\begin{aligned}
& f_{1}(q)=\left\|\frac{\operatorname{tg} \beta_{2} q h}{\operatorname{tg} \beta_{1} q h}-\frac{\left(\gamma_{1}+\beta_{1}\right)\left(c_{12}+c_{11} \beta_{2} \gamma_{2}\right)}{\left(\gamma_{2}+\beta_{2}\right)\left(c_{12}+c_{11} \beta_{1} \gamma_{1}\right)}\right\| \\
& f_{2}(q)=\left\|\frac{\operatorname{tg} \beta_{1} q h}{\operatorname{tg} \beta_{2} q h}-\frac{\left(\gamma_{1}+\beta_{1}\right)\left(c_{12}+c_{11} \beta_{2} \gamma_{2}\right)}{\left(\gamma_{2}+\beta_{2}\right)\left(c_{12}+c_{11} \beta_{1} \gamma_{1}\right)}\right\|
\end{aligned}
$$

Then mode discrimination was made from the found roots and group velocity of the Lamb waves were calculated.

But such luck solution of the system of equations (1), (2) occurs as written above in special case. In an arbitrary crystal direction of wave propagation, including (111) in whole, the system of equations (1), (2) isn't partitioned, and in this case we can not speak about "pure symmetric waves" and "pure antisymmetric waves", we have one intricate system of guide waves.

We calculated the frequency dispersion curves of phase $V_{\text {ph }}$ and group $V_{\text {gr velocities for Lamb mode }}$ propagation along $<100>$ direction in (100)-cut of $\mathrm{Cd}_{0.2} \mathrm{Hg}_{0.8} \mathrm{Te}$ plate. For the calculations, values of elastic moduli and density were taken from [10]. The curves for the lowest Lamb modes (for symmetric $s_{\mathrm{i}}$ and antisymmetric $-a_{\mathrm{i}}$, order $\left.i=0,1,2\right)$ are shown in Fig. 1a, b, accordingly. It can be seen that there are Lamb modes $s_{1}$ and $a_{2}$ characterized by existent of negative values of the group velocity near frequency of appearance. The negative sign of $V_{\mathrm{gr}}$ says that phase velocity have opposite direction as compared to the group velocity. Such waves are called as a reverse waves [5].

Such narrow range of existence of reverse Lamb waves and their super dispersion determine their supersensibility to alteration of medium elastic constants and finds its application in non-destructive testing of $\mathrm{Cd}_{0.2} \mathrm{Hg}_{0.8} \mathrm{Te}$ plates. 


\section{I.O.Lysiuk et al.: Properties and application of ultrasonic Lamb waves...}
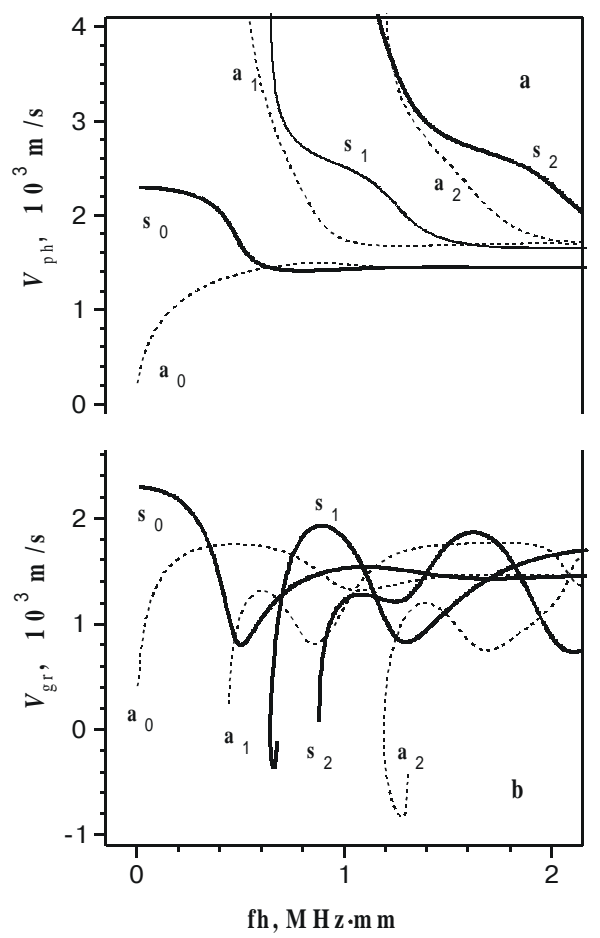

Fig. 1. Theoretical dispersion dependencies of ultrasonic Lamb mode velocities for propagation along $<100>$ direction in (100)cut of $\mathrm{Cd}_{0.2} \mathrm{Hg}_{0.8} \mathrm{Te}$ - plate: a) phase and b) group. Solid lines for symmetric $\left(s_{\mathrm{i}}\right)$ and dash - for antisymmetric $\left(a_{\mathrm{i}}\right)$ modes accordingly. The values of elastic modulus are given from [7].

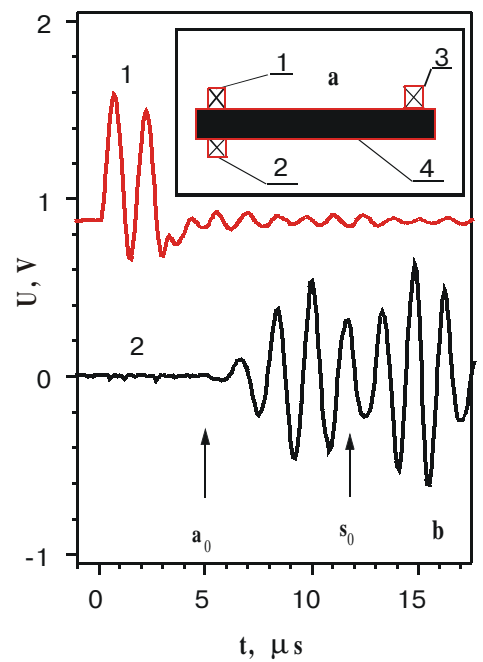

Fig. 2. a) The scheme of experiment is shown: piezoelectric transducers - 1,2,3; semiconductor sample - 4 .

b) Measured waveforms of pulse of excitation (on transducer 1, curve 1) and ultrasonic pulse passed across $\mathrm{Cd}_{0.2} \mathrm{Hg}_{0.8}$ Te-plate (111)-cut (on receiver transducer 3, curve 2) at frequency of $0.67 \mathrm{MHz}$. Curve 1 is shifted by $0.9 \mathrm{~V}$ and reduced by 10 times. The arrows correspond to arrival time of $a_{0}$ and $s_{0}$ mode s, accordingly.

\section{Exciting and measuring techniques}

The $\mathrm{n}$ - and p-types of $\mathrm{Cd}_{\mathrm{X}} \mathrm{Hg}_{1-\mathrm{X}} \mathrm{Te}$ plates $(x \approx 0.2$; square $S \approx 200-250 \mathrm{~mm}^{2}$; thickness $h \approx 1 \mathrm{~mm}$ ) were studied in the work. The previous measurements of velocity of bulk waves were carried out with ultrasonic interferometer continuous method in the range of frequencies from 0.5 to $10 \mathrm{MHz}$ with precision $2 \%$. The excitation of Lamb waves were performed using piezoelectric transducers in the form of bars made from ceramics PZT-19 polarized along thickness or width and $\mathrm{LiNbO}_{3}\left(\mathrm{Y}+36^{\circ}\right)$ - crystal cut. To measure group velocity $V_{\mathrm{gr}}$ and study efficiency of generation of Lamb waves, the pulse method was used. Modes were identified by comparison of experimentally measured $V_{\mathrm{gr}}$ with the predicted ones. Experimental $V_{\mathrm{gr}}$ values were determined by measurements of time delay of a passed ultrasound pulse across sample. The scheme of our experiment is shown in Fig. 2a. Two types of geometry of ultrasonic excitation were used: with one generating transducer 1 and double generating transducers 1 and 2. Transducer 3 served as a receiver.

Oscillograms of ultrasonic pulses were excited in (111)-cut of $\mathrm{Cd}_{\mathrm{X}} \mathrm{Hg}_{1-\mathrm{X}} \mathrm{Te}$ plates with one piezoelectric transducer and corresponded to vibrations along transducer width, and direction of Lamb wave propagation is shown in the Fig. 2b. The presence of two response pulses shows that two different modes of the Lamb waves were excited simultaneously and with approximate efficiency of excitement. In this example, the two modes can be separated, as they characterized by different time delays stemming from a difference in velocities of mode propagation. $\left(V_{\mathrm{s} 0}=0.8 \cdot 10^{3} \mathrm{~m} / \mathrm{s}, V_{\mathrm{a} 0} \approx 1.6 \cdot 10^{3} \mathrm{~m} / \mathrm{s}\right)$.

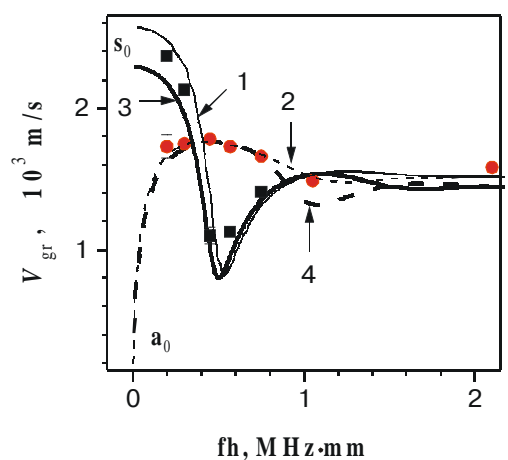

Fig. 3. Computed group velocities of lowest Lamb modes $\mathbf{s}_{0}$ and $a_{0}$ propagation in $\mathrm{Cd}_{0.2} \mathrm{Hg}_{0.8}$ Te-plate (100)-cut along $<100>$ direction (thick curves 1 and 2 ) and along $<110>$ direction (thin curves 3 and 4). Experimental sets of points in $\mathrm{Cd}_{0.2} \mathrm{Hg}_{0.8} \mathrm{Te}$ plate $(111)$-cut are shown as square $\left(s_{0}\right.$-mode $)$ and circle $\left(a_{0}\right.$ mode).

\section{Identification of Lamb waves in (111)- $\mathrm{Cd}_{0.2} \mathrm{Hg}_{0.8}$ Te plates}

The two experimental sets of $V_{\mathrm{gr}}$ for plate waves, which were measured for a $\mathrm{Cd}_{0.2} \mathrm{Hg}_{0.8}$ Te plate (111)-cut as frequency dependencies, are shown in Fig. 3 as solid squares 


\section{I.O.Lysiuk et al.: Properties and application of ultrasonic Lamb waves...}

and open circles, accordingly. It should be noted that comparison of results of measurements of these $V_{\mathrm{gr}}$ sets in several different directions of propagation for the specimen shows that within the range of experimental error $V_{\text {gr }}$ are coincided. Therefore, $V_{\text {gr }}$ in the plate studied exhibits isotopic behavior. Surely, this result is caused existence of sub block structure in studied crystals.

It is known [1-3] that orientation of blocks in $\mathrm{Cd}_{\mathrm{X}} \mathrm{Hg}_{1-\mathrm{X}} \mathrm{Te}$ crystals slightly changes by $1-3^{\circ}$ from one to another $[1,2]$ If density of blocks is in the range from 10 to $60 \mathrm{~cm}^{-1}$ along any direction of crystal length $(\approx 2 \mathrm{~cm})$ orientation of all subblocks can change by more than $60^{\circ}$, which corresponds to symmetry period of elastic properties of (111)-cut for plate of cubic crystals. Taking into account this aspect and the fact of a non-existent analytical solution of (1), (2) for (111)-plates, the comparison of the experimental data was made to identify them with theoretical curves of $V_{\mathrm{gr}}$ for $s_{0}$-and $a_{0}$ - modes propagation along [100]-direction and [110]-direction in (100)-cut of $\mathrm{Cd}_{0.2} \mathrm{Hg}_{0.8}$ Te plate (Fig. 3, thick curves 1, 2 and thin 3,4 , accordingly). Our experimental data find a good agreement with our assumption, which states that velocity of the lowest Lamb wave modes in (111) plane in $\mathrm{Cd}_{0.2} \mathrm{Hg}_{0.8}$ Te cut are close to $a_{0}$ and $s_{0}$ in (100) cut along $<100>,<110>$ directions (with precision $8 \%$.). As it could be seen from Fig. 3, the dispersion curve of velocity marked by squares are in the range between curves 1 and 2, which enables us to identify it as the velocity of $s_{0^{-}}$ mode. The fact that the dispersion curve marked by circles is close to curves 3 and 4, permits to consider it as $a_{0}$ mode.

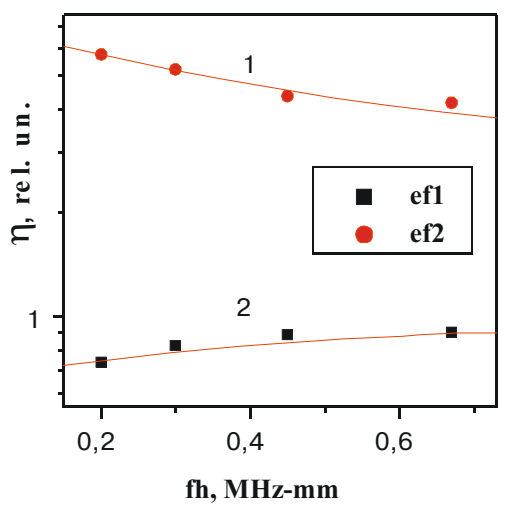

Fig. 4. Experimental frequency dependencies of relative efficiency of excitation $a_{0}$ mode with respect to $s_{0}$ mode $\eta=U a_{0} / U s_{0}$ in (111)-cut $\mathrm{Cd}_{0.2} \mathrm{Hg}_{0.8} \mathrm{Te}$ plate, where $U a_{0}, U s_{0}$, - amplitudes of $a_{0}, s_{0}$ modes. Curve 1 - geometry with one transducer 1 ; curve 2geometry with double-transducer 1 and 2 (see Fig. 2)

\section{Efficiency of ultrasonic mode generation and application of NWP}

From the viewpoint of application of the ultrasonic treatment of semiconductor plate, the efficiency of ultrasonic excitement in plate is one of the main parameters.
Relative efficiency of excitation $a_{0}$-mode with respect to $s_{0}$ mode $\left(\eta=U a_{0} / U s_{0}\right)$ in the frequency range from 0.2 to $2 \mathrm{MHz}$ has been studied, where $U a_{0}, U s_{0}$, amplitudes of $a_{0}, s_{0}$ modes. Curve 1 in Fig. 4 shows that efficiency of generation of $a_{0}$-mode with one piezoelectric transducer is about 5 times higher than the efficiency of $s_{0}$-mode generation. Curve 2 in Fig. 4 and oscillogram curve 2 in Fig. 2 exhibits approximately equal efficiency of the Lamb mode generation with two piezoelectric transducers. Fulfilled researches have led to conclusions: the method of attached transducers enabled to excite the lowest Lamb modes $\left(a_{0}\right.$ and $\left.s_{0}\right)$ simply and reliably; the usage of double -transducer improves the efficiency of generation $s_{0}$ mode; the greatest relative efficiency of excitation of the $a_{0}$ Lamb mode $(\eta=5.7)$ is achieved with one transducer generation at frequency of $f h \approx 0.2 \mathrm{MHz} \cdot \mathrm{mm}$.

The Lamb waves are conventionally used in non-destructive testing of plates [11]. For this application the $a_{0}$ and $s_{0}$ modes are proposed. As shown in Fig. $1 \mathrm{~b}$, the antisimmetrical $a_{0}$ is very sensitive to the thickness of plate if $f h<1 \mathrm{MHz}$ mm holds. As $a_{0}$ has low velocity of propagation, which increase accuracy of defect location detection, it can be applied for nondestructive testing. The dispersion of the symmetrical $s_{0}$ mode if $f h<0.25$ $\mathrm{MHz} \mathrm{mm}$ holds is negligible so it can be used for defect location in plate with slight non-parallel side. $s_{1}$-Lamb mode in the range of $0.64-0.68 \mathrm{MHz}-\mathrm{mm}$ and $a_{2}$ in the range 1.19-1.31 MHz-mm are reverse Lamb waves and can be used for non-destructive testing $\mathrm{Cd}_{0.2} \mathrm{Hg}_{0.8}$ Te plate which enables us to check uniformity of material.

\section{Conclusions}

In the paper, the frequency dispersion curves of phase and group velocities for Lamb mode propagation along $<100>$ and $<110>$ directions in (100)cut of $\mathrm{Cd}_{0.2} \mathrm{Hg}_{0.8} \mathrm{Te}$ plate are obtained. The methods of generation of specific NWP modes were elaborated; the group velocity $V_{\text {gr }}$ was measured in range (0.5-10) $\mathrm{MHz}$ in (111)-cut of $\mathrm{Cd}_{0.2} \mathrm{Hg}_{0.8} \mathrm{Te}$ plate; the lowest $a_{0}$ and $s_{0}$ Lamb wave modes were identified. The comparative study of the generation efficiency of separate modes provides conclusion that $a_{0}$-mode has much more efficiency of generation than the $s_{0}$-Lamb mode and may be recommended for ultrasonic treatment of $\mathrm{Cd}_{0.2} \mathrm{Hg}_{0.8}$ Te samples. Some features of the Lamb wave modes for ultrasonic diagnostics are discussed.

Taking into account that investigated materials are widely used in infrared engineering in the form of heterostructures and layered structures $\mathrm{CdHgTe/}$ $\mathrm{CdTe}$, there is the necessity in special researches of the acoustic characteristics of NWP in such objects. 


\section{I.O.Lysiuk et al.: Properties and application of ultrasonic Lamb waves...}

\section{References}

1. A.V. Lubchenko, Ya.M. Olikh, K.A. Myslyvets. Rekombinatsiya nositeley cherez aktseptornye urovni sobstvennykh defektov n- $\mathrm{Cd}_{\mathrm{X}} \mathrm{Hg}_{1-\mathrm{X}} \mathrm{Te}$, podvergnutykh UZ obrabotke. // Fizika i texnika poluprovodnikov, 24, p.271273 (1990)

2. Ya.M. Olikh and Yu.N. Shavlyuk. Acoustically stimulated suppression of 1/f noise in subblok CdHgTe crystals // Sov. Phys. Solid State, 38 (11), p.1835-1838 (1996).

3. Ya.M. Olikh, R.K, Savkina, O.I. Vlasenko. Acoustostimulated activation of bond defects in CdHgTe alloys // Semiconductors 33, p.398-401 (1999)

4. S.W. Wenzel and R.M. White. A multisensor employing an ultrasonic Lamb-wave oscillator // IEEE Trans. Electron Devices, 35, p.735-743 (1988)

5. I.V. Ostrovskij, // Akustoluminescenciya i defekty v krystalakh, Vyshcha shkola, Kiev 1995, p. 62-83 (in Russian).
6. S.S. Ostapenko and I.H. Tarasov. Nonlinear resonance ultrasonic vibration in Czochralski-silicon wafers // Appl. Phys. Lett., 76, pp. 2217-2219 (2000).

7. Yu.V. Khalak, Amplitude-dependent ultrasound attenuation due to inclined dislocations // Condensed. Matter. Physics. N10, p.61-66 (1997).

8. I.A.Victorov. Rayleigh and Lamb Waves: Applications, Plenum, New York, 1978.

9. L.G. Merkulov, D.A. Tursunov. Phase velocities of normal waves in a crystal cubic plate // Akusticheskiy zhurnal, 1, p. 136-138 (1969). (in Russian)

10. I.O. Lysiuk, V.F. Machulin, Ya.M. Olikh. Anisotropy of ultrasonic wave propagation velocities in $\mathrm{CdHgTe} / \mathrm{CdTe} / /$ Semiconductor Physics, Quantum Electronics and Optoelectronics 2, pp. 28-30 (1999).

11. J.L. Rose. Guided Wave Nuances for Ultrasonic Nondectructive Evaluation // IEEE Trans. Ultrason., Ferroelect., Freq. Contr 47, pp. 575-583 (2000). 